\title{
Striatal hypermetabolism in a case of IgG4-related disease
}

\begin{abstract}
Abdelhamid Biyi, Omar Ait Sahel, Abderrahim Mejjad, Yasser Benameur, Salah Nabih Oueriagli, Abderrahim Doudouh Mohammed V University of Rabat, Department of Nuclear Medicine, Mohammed V Military Teaching Hospital, Mohammed V University of Rabat, Rabat, Morocco
\end{abstract}

[Received 27 XII 2017; Accepted 14 III 2018]

\begin{abstract}
A 77 years-old man with lung and bone involvement of proven lgG4-related disease complained of tree month's memory deficits. Brain MRI was normal. 18F-FDG whole body PET/CT showed in addition to the bone lesions, a marked symmetrical striatal hypermetabolism in contrast with cortical hypometabolism. Despite steroid treatment, the patient's clinical status declined rapidly and he died two months later. Striatal hypermetabolism has been reported in autoimmune limbic encephalitis, but to the best of our knowledge, this is the first description in a case of IgG4-related disease.
\end{abstract}

KEY words: IgG4-related disease, striatal hypermetabolism, limbic encephalitis, 18F-FDG PET/CT

Nucl Med Rev 2018; 21, 2: 109-110

Immunoglobulin G4 related disease (IgG4-RD) is a multi systemic disorder which was first described in 2003 in patients with autoimmune pancreatitis. It is characterized by a dense lymphoplasmocytic infiltrate rich in IgG4 plasma cells and storiform fibrosis in almost every organ. The most common are pancreas, salivary glands, liver, biliary tree, orbit, kidney, pericardium, aorta, lung, breast and prostate. The central nervous system is more rarely involved. When it occurs, hypertrophic pachymeningitis and pituitary thickening are the most frequently reported.

Here, we report a case of 77 years-old man who complained of tree month's headache, right face pain, and more recently memory deficits and temporospatial disorientation. No signs of systemic sepsis were evident. CT scan showed a large lytic area on the left orbit and zygomatic process, the clivius, and the $1^{\text {st }}$ and $2^{\text {nd }}$ cervical vertebrae. Brain MRI was normal. The cerebrospinal fluid was clear, acellular, and had normal protein levels. As malignancy was suspected, a bone scan was performed and showed multiple hot spots over the same lesions as those mentioned above. Serum tumor markers and auto-antibodies' (ANA, Anti-ECT, ANCA) were negative. Serum level of IgG4 was elevated $(2,290$ $\mathrm{g} / \mathrm{l}$, normal range: 0.040 à $0.870 \mathrm{~g} / \mathrm{l}$ ) and immunohistochemical study of the right zygomatic process lesion revealed increased number of IgG4-positive staining plasma cells up to 30 per high powered field. We consequently performed an 18F-FDG whole body PET/CT as a part of the initial process of an IgG4-RD. In addition to the bone lesions (Figure 1), there was a hypermetabolic areas in the upper lobe of the right lung, and marked symmetrical

Correspondence to: Abdelhamid BIYI, M.D.

Department of Nuclear Medicine, Mohammed V Military Hospital of Rabat, Mohammed V University of Rabat; P Box 6614 Rabat Instituts, Morocco; e-mail: abdelhamidbiyi@yahoo.fr striatal hypermetabolism in contrast with cortical hypometabolism (Figure 2). Intravenous steroid treatment was started but the patient's clinical status declined rapidly. He died two months later.

Limbic encephalitis is usually associated with autoimmune diseases, viral infections and malignant tumors as a paraneoplastic syndrome. In IgG4-RD, few cases of "encephalopathy"

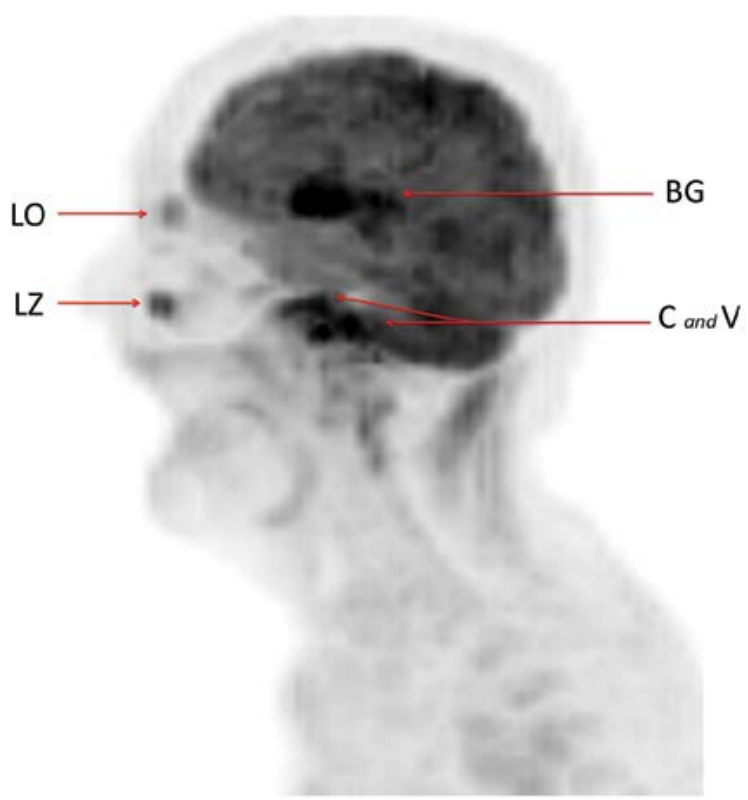

Figure 1. Maximum intensity projection PET image (in left projection) revealing 18F-FDG avid bone lesions on the left orbit (LO) and zygomatic process (LZ), the clivius $(C)$, and the $1^{\text {st }}$ and $2^{\text {nd }}$ cervical vertebrae (V) along with intense hypermetabolism in basal ganglia (BG) 
A

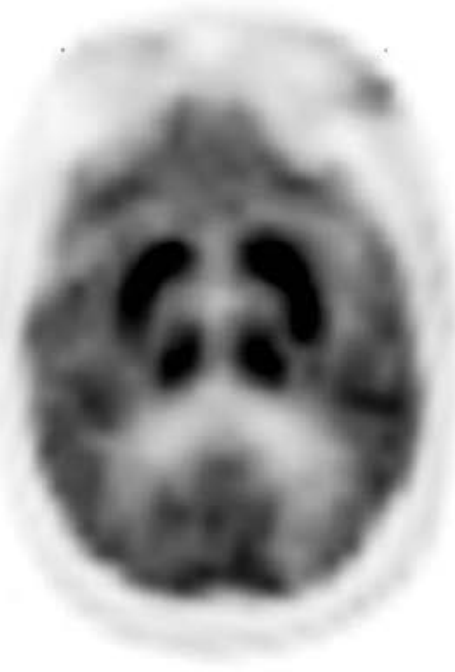

B

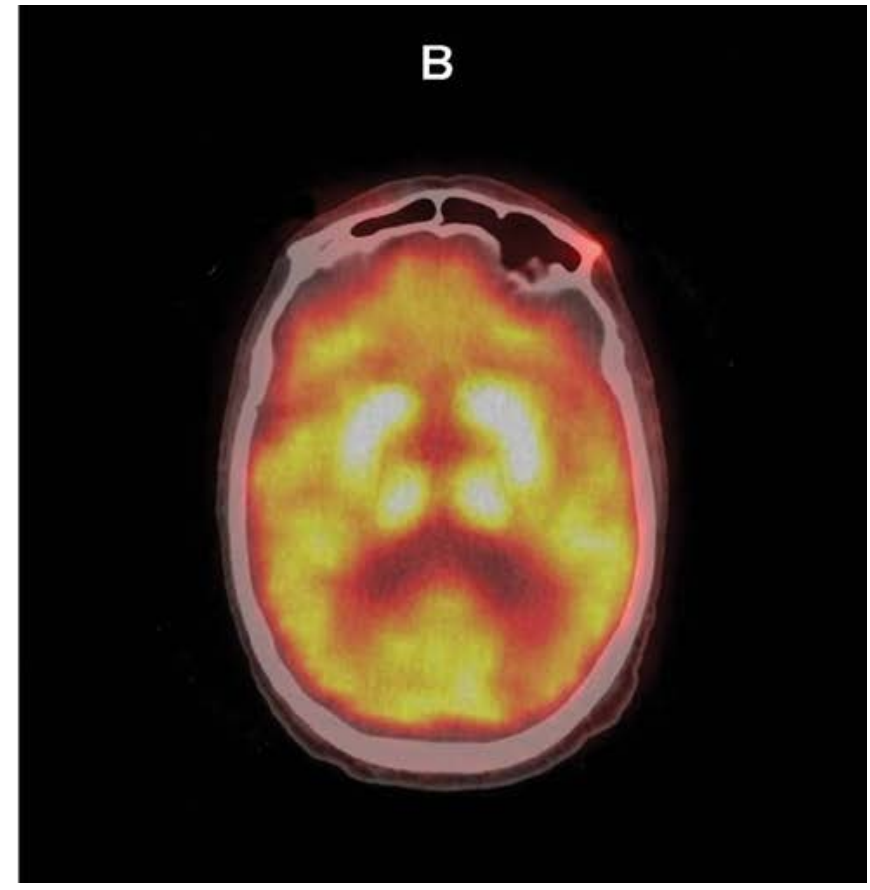

Figure 2. 18F-FDG PET (A) and fusion (B) brain transaxial images showing intense and symmetric hypermetabolism in basal ganglia in contrast with marked cortical hypometabolism

with a focus on MRI patterns and significant mortality have been reported [2]. In our patient, even if no post-mortem was performed, 18F-FDG PET findings corroborated neurological symptoms. Striatal hypermetabolism on 18F-FDG PET scan has been previously reported in autoimmune limbic encephalitis [3] but, to the best of our knowledge, this is the first description in lgG4 related disease. Further studies are needed to elucidate its mechanism and prognostic significance.

\section{References}

1. Kamisawa T, Funata N, Hayashi $Y$, et al. A new clinicopathological entity of IgG4-related autoimmune disease. J Gastroenterol. 2003; 38(10): 982-984, doi: 10.1007/s00535-003-1175-y, indexed in Pubmed: 14614606.

2. Heine J, Prüss H, Bartsch T, et al. Imaging of autoimmune encephalitis-Relevance for clinical practice and hippocampal function. Neuroscience. 2015; 309: 68-83, doi: 10.1016/j.neuroscience.2015.05.037, indexed in Pubmed: 26012492.

3. Rey C, Koric L, Guedj E, et al. Striatal hypermetabolism in limbic encephalitis. J Neurol. 2012; 259(6): 1106-1110, doi: 10.1007/s00415-011-6308-2, indexed in Pubmed: 22081102. 